\title{
Interpreting fitness: self-tracking with fitness apps through a postphenomenology lens
}

\author{
Elise Li Zheng ${ }^{1}$
}

Received: 6 August 2020 / Accepted: 12 January 2021

(๖) The Author(s), under exclusive licence to Springer-Verlag London Ltd. part of Springer Nature 2021

\begin{abstract}
Fitness apps on mobile devices are gaining popularity, as more people are engaging in self-tracking activities to record their status of fitness and exercise routines. These technologies also evolved from simply recording steps and offering exercise suggestions to an integrated lifestyle guide for physical wellbeing, thus exemplify a new era of "quantified self" in the context of health as individual responsibility. There is a considerable amount of literature in science, technology and society (STS) studies looking at this phenomenon from different perspectives, linking it with the sociology of self-surveillance and neoliberal regimes of health. However, the human-technology interface, through which the micro- (behavioral) and macro- (social) aspects converge, still calls for extensive examination. This paper approaches this topic from the postphenomenological perspective, in combination with empirical studies of design analysis and interviews of fitness apps, to reveal the humantechnology link between the design elements and people's perception through the direct experiences and interpretations of technology. It argues that the intentionality of self-tracking fitness app designs mediates the human-technology relations by "guiding" people into a quantified knowledge regime. It shapes the perceptions of fitness and health with representations of meanings about a "good life" of individual success and management. This paper also gives a critique of current individual, performance-oriented fitness app designs and offers the possibility of seeking alternatives through the multistable nature of human-technology relations-how altering interpretation and meaning of the design with a cultural or social context could change the form of technological embodiment.
\end{abstract}

Keywords Postphenomenology $\cdot$ Self-tracking $\cdot$ Fitness apps

\section{Introduction}

The spring of 2020 is certainly not the best time to take exercises-the global pandemic COVID-19 has trapped millions of people across the world at home (especially those told to self-quarantine). Gyms are closed; running or walking outside is not encouraged; there is certainly no team sports due to social distancing; Those who would like to stay active have to turn to in-door alternatives like fitness apps. "Making your home your own gym," claimed a popular Chinese fitness app "Keep" on one of their campaigns during the coronavirus outbreak. ${ }^{1}$ However, for those who desperately want to keep their shape tuned during a difficult

Elise Li Zheng

elise901@gatech.edu

1 Department of History and Sociology of Science and Technology, Georgia Institute of Technology, 221 Bobby Dodd Way, Atlanta, GA 30309, USA time, another well-known slogan from "Keep" is much more needed to progress during isolation when everyone is trapped indoors-"Self-discipline brings freedom."2

Fitness apps have gained popularity with widely available mobile personal technology like smartphones and wristbands, years before the COVID-19 outbreak. These "gigeconomy" innovations focus on the consumer's market and correspond with self-tracking and self-management trends, promoting extensive engagement through interactive design elements. With these innovations, fitness apps have revealed various intriguing façades of health and fitness in our daily life. For those who want to exercise everywhere and keep track of their fitness status, fitness apps can easily help users be physically active anywhere and everywhere. The apps help manage their daily physical exercise, movement

\footnotetext{
${ }^{1}$ Promotional message retrieved in the "Discovery" and "Community" section (where users can find online campaigns or exercise challenges to complete) of "Keep" Fitness App, February 2020.

2 This message shows up every time a user opens the Keep App.
} 
or even dieting regiment, summarizing one's fitness status with quantified data charts (such as the MyFitnessPal in the US, "Keep" in China). Alongside these "at-home," convenient exercise guides and features, there is also a sense of dedication: one should always count on the tracking records of calories and exercising time to keep fit. Besides, fitness apps also gamify fitness exercise, orienting the way of using it into a "fun" setting (e.g., Nintendo "Ring-Fit," released in 2019, which turns exercise into adventure games). They sometimes turn everyday actions like walking into a numerical competition with your friends (e.g. "WeChat Exercises" in China, see Gui et al. 2017), adding social network elements into the management of health.

Emerging technologies have changed the way people exercise. But do these new trends change the way people see health and fitness? More importantly, as the trending technologies incorporate new elements of self-exercising and self-management, how do they correspond with the concepts and understandings of personal responsibility and personal health? If so, what is the role of technology in this process of persuading people to do certain things or to subscribe to certain knowledge regimes? What is the technical and social mechanism behind those changes? Most importantly, as more studies start to examine the social implications of various gadgets, how are the social messages embedded in technological designs and delivered through interactions?

In this paper, I will use some concepts from the philosophical perspective of postphenomenology to explain the technologically mediated relationships between fitness app users and health/fitness practice. Based on the empirical case analysis of a Chinese fitness app, I argue that the technological intentionality embedded in the interface design gives users a normalized view of fitness and guides them to perform an extensive engagement with personal data they have generated during exercise. The design elements also help users to create the hermeneutic interpretation of achievement as the core elements of "keeping fit." However, the social and cultural context of fitness allows different users to develop a "multistable" relationship with the app.

\section{Monitoring health as a "self-centered" practice}

A growing body of literature has paid attention to fitness apps and self-tracking. The studies range from the sociopolitical analysis that draws upon Foucauldian theories of self-surveillance and governmentality, socio-psychological and behavioral studies that focus on the effectiveness of health promotion, to Science, Technology and Society (STS) studies that focus on human-tech interactions. Sociologists criticize this "quantified self" trend as a neoliberal form of self-governance, which has reduced the social context of health to a bunch of numbers with an increasing focus on individual responsibility and self-surveillance (Ajana 2013; Lupton 2012 and 2016; Neff and Nafus 2016; Ruffino 2018). Some of them raise questions about the politics of bodily control in public and private life (Lupton 2016) and the value, agency, and expression of identity (Ajana 2018). As Foucault (Foucault 1988 and 1990) has argued, technology is the manifestation of the materialized, normalized surveillance power, which works through the internalization of certain values and ethics onto the discipline of self. It perpetuates through everyday engagement with technology. In this way, the status of health and fitness, constructed through a series of numbers with tracking data, reflects the way people see themselves: as "objective self-fashioning" (Dumit 2003) or "technoscientific identity" (Clarke et al. 2003; Rabinow 2010; Rowse 2015). The digital data circulates as a new form of knowledge and shapes the meaning of a socially accepted "self" (Lupton 2016) —in this case, a healthy, welldisciplined self.

Looking from the socioeconomic perspective, the prevalence of self-surveillance technology not only puts the responsibility on individuals but also interprets fitness as commodities (Clarke et al. 2003; Millington 2016; Alam 2016). The "datafication of everything" process is based on collecting and valuing personal data, which generates business opportunities (Millington and Millington 2015; Millington 2016). Thus the automated fitness trend unifies self-identity, commerciality and neoliberal healthism at the same time with problems of data privacy and ownership controversies (Ajana 2017).

On the other hand, the micro-perspective of human-technological interactions opens channels of new observations on the behavioral side. The nonhuman elements mediate the dynamic and experience of using technology. Research shows that self-tracking technology can facilitate learning insights about one's body and mind (Choe et al. 2014); function as triggers for action (Ajana 2017); give a "nudge;" (Lupton 2014) or play the role of an authority that shows the persuasive effect (Fogg 2002). Users also divert themselves from the projection of technology, finding meanings or engaging in negotiations (Ruckenstein and Schüll 2017; Lomborg et al. 2018; Didžiokaitè et al. 2018).

The nuances in the textual contents and designs of fitness apps call for further investigation-how they are connected with the wider social, cultural and political pictures via an individual's interactions and experiences, where the socially constructed "self" is embodied. This paper aims at explaining the link between personal technology and constructed perception of health at the level of technological interface-What does this process look like? How do people experience, feel and perceive the technological design and subsequently build meanings and understandings of fitness and health in a social context? How can this connection 
have implications for engagement and design? Most current sociological studies are falling short of revealing the interactive mechanisms where the conversations between people and technology occur. Technologies are treated as something static and "external." As Lupton (2019) recently calls for a "more-than-human" perspective between technology and users, the interactions need to be examined in a more dynamic and "internal" ${ }^{3}$ way, and a closer investigation on the phenomenal of human-technology connections is needed. Thus, a postphenomenological perspective of technology is introduced.

\section{Postphenomenology: a way of closer investigation}

In the fields of STS, a philosophical theory of postphenomenology has offered enlightening perspectives. This empirical-rich, versatile theory that combines phenomenology and American pragmatism has been widely adopted in the studies of human-technology relationships. According to Rosenberger and Verbeek (2015), these studies focus on the various ways in which technology helps to shape the relations between humans and the world via its "mediation," which plays not only an instrumental role but is closely intertwined with human experiences and practices. Researchers have closely examined human actions and ideas about technological incorporation by analyzing technology-related experiences and meanings, revealing the shaping power of such technology, especially the interface upon which the technology and human connect and interact (such as designs, visualization image, etc.).

In this paper, I will use a few terms and concepts that postphenomenologists have coined and applied in delineating the interactive process between human and technology ${ }^{4}$ :

(1) The "intentionality" of artifacts, which is embedded in the technological interfaces that "tend to" guide, interrupt or form behavioral habits of humans (Ver-

\footnotetext{
${ }^{3}$ By saying "internal," I don't mean that the technology is part of our somatic bodies (although in some cases, the technology cannot be separated from it, such as a cardiac pacemaker). Humans are increasingly utilize technologies as a way of seeing/understanding the world and reflecting on themselves, whether or not a certain technology is attached to them at all times.

${ }^{4}$ There are some other forms of mediating relations, namely "embodiment" relations, in which technology broadening human's sensitivity and human look "through" them to recognize and connect with the material world (Verbeek 2005: 125). Also, there are two other mediating relations that I will not elaborate in this paper: alterity (technology as a quasi-other, such as robot) and background (not consciously experienced but shaping the environment). See Rosenberger and Verbeek 2015
}

beek 2005). It studies how the appearances of technology (such as interactive interfaces) bridge the subjects (human) and objects (technology) during the interactions. It can indicate how technologies can be directed at particular aspects of reality and the "purposiveness" that technologies can embody (Verbeek 2011). The relationship of human and technology shapes itself in the joint interaction between technology and human, "adding technological intentionality to human intentionality." (Ibid: 145)

(2) The "hermeneutic" relations. In this way, technology "explains" the world through interactive designs and experiences, which helps to form the perceptions of the world. Meanwhile, the materiality of technology provides a representation of the world to the human, and the human develops an interpretation of it (Verbeek 2005). Certain forms of technological intentionality would direct people into certain hermeneutic interpretations.

(3) "Multistability." The term "multistability" refers to the way users may interpret different meanings during technological interactions or create different bodily experiences under different scenarios and contexts. ${ }^{5}$ Also, the interaction between artifacts and humans is not intrinsic nor deterministic. It bears different forms in different contexts, against a totalizing narrative. The process of forming multistable relations-even for the most mundane objects-uncovers new information or re-interprets cases in productive ways (Rosenberger and Verbeek 2015; Rosenberger 2017a) and calls for a more flexible and contextual approach to studying the technology. For example, according to Tripathi (2017), technologies are culturally multistable and open to a culturally embedded "hermeneutic act" during human interaction.

\subsection{Technological mediation, self-tracking and fitness}

Several postphenomenology scholars have researched selftracking, focusing on both theoretical implications (Ihde 2002; Kristensen and Prigge 2018; Kristensen and Ruckenstein 2018) and empirical investigations (Van Den Eede 2015; Secomandi 2017 and 2018). The self-tracking practices have contributed to a constructed, culturally relevant image of the health and fitness of human bodies (Van Den Eede 2015). All this research has shown the role of design to be an important nonhuman factor in technological mediations, and the "intentionality" of technology offers the

\footnotetext{
5 "Stability" or "variation", see Rosenberger and Verbeek 2015: $25-26$.
} 
possibility to interfere, intervene or even interrupt our life. Meanwhile, the mediation takes many forms ("multistability") that call for further investigations.

The "embodiment" aspect of self-tracking devices has altered the user's bodily perceptions. The experiences and practices of "bodily skills" count as a form of "human technique," guiding and shaping our actions from the "bodies in technologies." (Ihde 2002) For example, the tracking results have rendered our physical health and fitness status visible and intelligible with numbers and metrics, which are easily connected with an academic-based knowledge regime and transfer the sense of a somatic self from "Body One (our own, physical body)" to "Body Two (A socially and culturally intelligible construction)." (Ihde 2002) The use of electronic health records can reinforce the embodiment by "fading into the background, becoming a means of experience." (Moerenhout et al. 2019) Besides, the "hermeneutic" mediations of tracking devices translate bodily performances and shape the user's perception of health and fitness status. The making of "body image" (Ihde 2009) here represents the knowledge production process.

Secomandi (2018) analyzes the technological mediation of a commercial tracking device (DirectLife) as a "service interface," where the human-technology interaction reveals a "reference and translation issue." (Ibid: 88 ) The process includes not only the users but also the maker's design dynamics, which also shapes the entire technological experience (Secomandi 2017). Self-tracking devices make present the objective body as a site for hermeneutic inquiry, creating the link between subjective and objective body (de Boer 2020) as we read numbers, reflect and interact with them.

The postphenomenology perspective is not only rich in theoretical constructions but also methodological implications. It bounds the micro-and macro- aspects of technological design, accounting for cultural and social factors in the direct phenomenon of the user's interaction with the interface. It offers possibilities of connecting neoliberal health regimes and culture of self-surveillance with design elements in a market-driven network. For sociologists, it is another way to address the Foucauldian thoughts: a materiality-based power structure casting upon a self-practice (Verbeek 2011). The approach can better articulate human experiences with a sense that the technologies comprise a critical part of how people feel, sense and comprehend the world, thus building human-technology connections more dynamically and organically. Moreover, the philosophy-rich fields need to be combined with more case studies on the connection between technology and people's health in daily life.

\section{Case study: interviewing fitness app users in China}

To examine the empirical implications of postphenomenology on the fitness apps, I will use the case study of the most popular fitness app in China, "Keep," It was first released in 2015. Since then, it has gained notable popularity among the urban, middle-class population in China. It claims to have over 100 million installations across the Chinese smartphone market, with monthly active users totaling over several million. It offers home training sessions and tutorials that people can follow and can help users keep track of their activity levels, lengths of time and calories burnt, among many other features that users could engage in. The product description addresses its convenience and accessibility of the training sessions and various options to fulfill needs from beginners to an advanced fitness level. It would walk the users through the process with extensive, everyday engagement-almost free (unless you're lured into buying their premium services). At the same time, it tries to motivate users with its inspirational slogans of self-discipline (such as "self-discipline brings freedom") and virtual rewards from continuous tracking, monitoring, reflecting and sharing of their fitness status.

But how is the self-tracked fitness ideal carried out among users, and how do users consume the messages that the App provides? Do they self-identify as self-disciplined, motivated individuals during the interaction with the technology user-friendly design? To investigate the experience of using the App, I have collected a sample of 20 users online from social media platforms as well as my personal social network (which is not a very hard task thanks to its popularity) and conducted semi-structured interviews. ${ }^{6}$ They were between 20 and 45 years old and consisted of 9 males and 11 females, all from urban areas in China. Their frequency of use and level of engagement of the App ranged from occasional or casual users to dedicated or long-term trackers. The interviews covered questions of habits, preferences, behaviors and "feelings" (such as "how do you feel when you use certain features" and "how do you like its interface") with the App. The interviews asked their understanding of fitness and health through digesting the messages and contents of the App. The analysis is built on a thematic basis-to generate meanings and interpretations by coding and categorization with the help of social theories (Mayring 2004; Charmaz 2006). In this paper, I will focus on the user's interpretation of meanings about health, personal life and achievements associated with technological designs and then apply and

\footnotetext{
6 The interviews were conducted in the summer of 2019, and some following-ups in the spring of 2020.
} 
develop theoretical insight according to the analysis of these empirical materials.

\section{1 "Flow" and "follow": intentionality behind normalization and engagement}

Upon registration with the App, the user will enter his/ her (biological) sex, age and body measurements (such as weight and height), then run through a self-evaluation of "fitness level" before getting a "personalized" plan for everyday exercises. It will determine the "need" for different levels and types of workouts, such as "losing weight," "keeping fit," or "toning up muscles." The App will then recommend the user a series of exercise routines or give the user a monthly workout plan based on the "fitness level" and goals. The entire process specifies each exercise routines, length of time, purpose (e.g., which part of body one set of routine is supposed to work on), and the calories it will burn. The user is supposed to follow the recommendations and to earn progress toward the goal.

As sociological critics indicate, the user's "body" here is supposed to meet a standardized norm and be placed onto a linear diagram of fitness (the "fitness level") and decontextualized in a way that objectifies body parts as targets for intervention. It serves as the "embodiment of quantification of self" (Lupton 2016) and the initial intention of one to monitor the status of fitness to comply with a specific standard, complying with the belief that universality of science could contribute to the health of bodies corresponds with Foucault's view of surveillance and norms (Foucault 2012).

However, the intentionality of the interactive design gives the user a different impression of normalization. As Ihde (1990) proposes, the intentionality of technology bridges up the human-technology - world arch and mediates the relations into certain forms and trajectories, ${ }^{7}$ and the specific ways in which specific technologies can be directed at specific aspects of reality (Verbeek 2008). Here, technology interferes with the user's life by offering "guidance" through this process and selects certain information for a simple, user-friendly display. As Ihde (1990) and Verbeek (2005) identify as an "innate trajectory," the intentionality becomes visible through the promotion of the designed "flow." It is shared by other user-centered fitness apps and games apps and games which tend to make "immersive" and "effortless" experiences tailored for specific users (Maloney et al. 2015; Martin and Kluckner 2014). Users follow the designed process as a proper, "scientific" guidance. It will develop a positive feedback loop when the users meet the norms and

\footnotetext{
7 This can also be traced back to Heidegger's "Instrumental Intentionality" that something's "in-order-to" feature becomes the context of the thing that "could be done." See Verbeek 2001.
}

standards by getting rewarded or upgraded, and in this way, they comply with the norms of regulating the bodies.

To the users, the initial process-a series of clicks and filling in blanks that lead to a visualized display-makes intelligible communications of "which level am I at?" "How fit am I?" and "What should I do to improve?" Much of the information about "what to do" (such as time of exercise, intensity, and exercise goals) is easily accessible in a conversational style and mostly visible throughout the experiences. Users connect themselves with the numbers they need to achieve, according to which level they are assigned and which three goals they choose. The intentionality of the "flow" prioritizes accessibility and simplifies decisions, while a majority of users describe their experiences as "easy" and "clear." For this reason, although packed with information and numbers, the design style of the App is sleek and simple to serve many fitness beginners for intentionality towards easy use and simple comprehension, and which "directs" users into thinking fitness in the same way with the design languages. For example, one user stated,

"I think [the evaluation] is quite appropriate. I would feel challenging when I finish about $2 / 3$ length of the exercise, but I think it is normal [to feel that way] ...it makes an evaluation based on this, you have to put yourself into it to get an accurate outcome." (\#2, female, in her $20 \mathrm{~s}$ ).

"It is convenient...not so many steps, and loads of recommendations...(The exercise tips) show up timely before and after exercise, and I'll click on to read, sort of a 'no-brainer' reading without a hitch." (\#19, female, in her $20 \mathrm{~s}$ ).

Also, the notification system with sound effects enhances the intentionality, giving the app the impression of some kind of "guidance." Thus, it is also easy to subject oneself to the App's framework of evaluating "standard" human bodies. Users take in the information without much of the impediments of fitness-related jargon.

"[When I follow the exercise instruction during exercise, the video guide] will shout out to 'keep up' just the right time when I feel tired." (\#4, female, in her $30 \mathrm{~s}$.)

"I've switched off all the notification on my phone other than Keep. It'll send a notification to me to remind me to exercise; I hope it could work." (\#4, female, in her $30 \mathrm{~s}$.)

The intentionality of design is meant to be "followed." The users are supposed to be consuming the App's information when needing assistance, instructions and reminders to keep fit. Users respond to the intentionality of design with simpler, routinely choreographed patterns, such as following notifications and personalized plans (which include just 


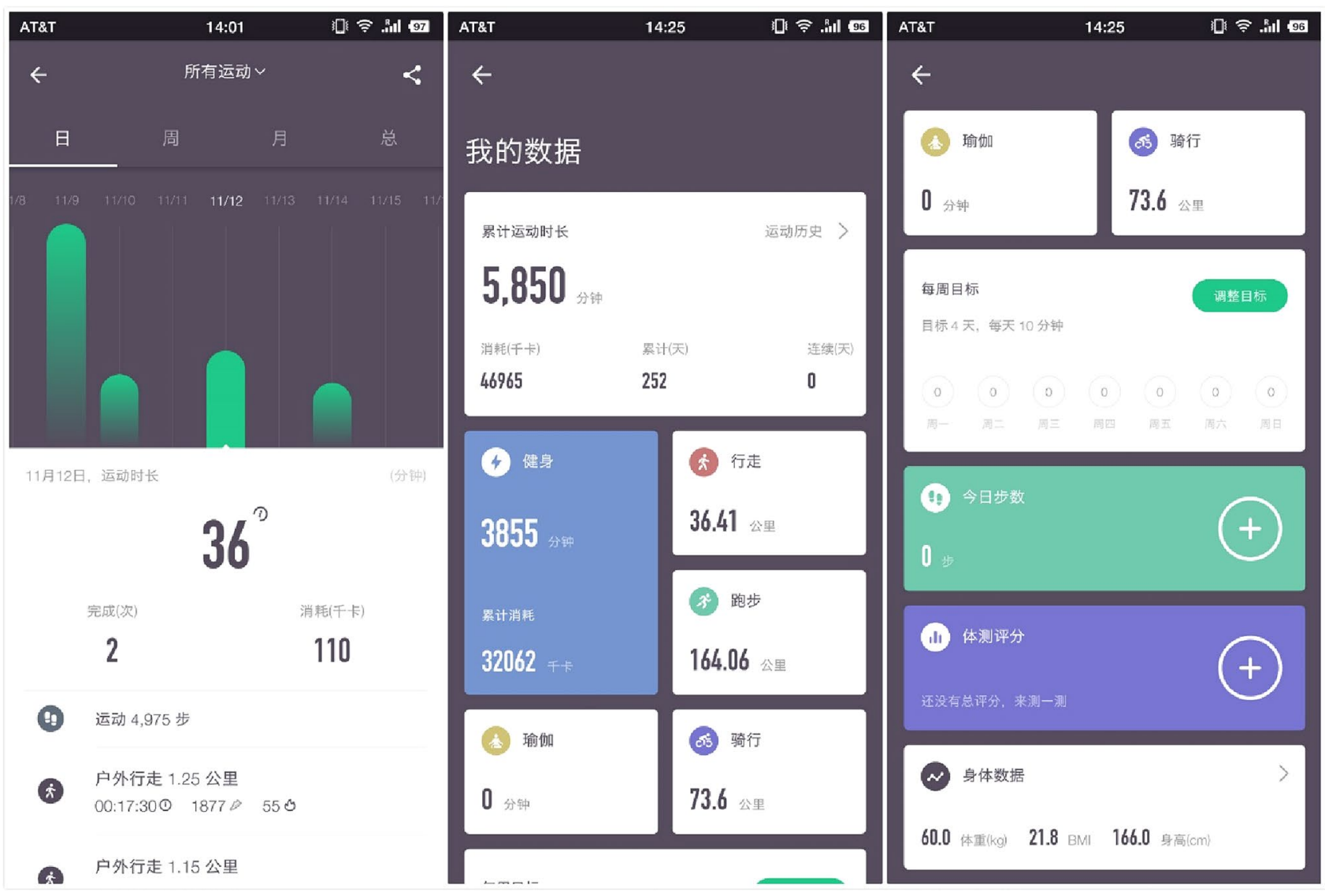

Fig. 1 Diagrams of fitness achievement. The left shows the daily/ weekly/monthly summary of exercising time. It shows that the user (the author) has done $36 \mathrm{~min}$ of exercise in two routines on this particular day, burning $110 \mathrm{cal}$. In the middle, it shows "My Data," with a myriad of different types of exercise (Starting from the left blue box counterclockwise): total time, fitness routines, Yoga, walking,

a few exercises). Users generally embrace the "no-brainer" of design and information display. However, during this process, more subtle personal needs are sacrificed for a pre-determined, easily applicable setting that a popular app needs to develop a wider user base. Other social determinants of health, such as access to healthy food, built environment and socioeconomic status and mental status, are ignored in the initial design and narrow the scope of equating "healthy-life" to self-disciplined practices that could be translated into numbers. The idea that a "healthy life" must be quantified to count has reduced health and fitness onto a thin layer of workouts and engagements.

\subsection{Engagement and achievement: intentionality and hermeneutic relations}

Another design component that bears intentionality is the emphasis on engagement. The App gives considerable emphasis on "discipline" and "management"—exercising running, cycling. On the right, it continues with a weekly chart-if you have exercised, a circle will be filled, followed by the walk steps (the green box, where the number is 0 ), fitness score (the purple box, linked to Fig. 1 if the user clicks on it) and bodily metrics (here shows body weight, BMI and height)

every day, monitoring every movement, sharing with friends and families, and increasing overall activity levels so that one could be rewarded with a virtual upgrade of his/her user status. All of these are to prompt engagement with various functions within the App. For a commercially available app, the aim is to expand the user base as well as to boost the engagement level. The MAU (monthly active users) and DAU (daily active users) are two of the most important metrics for evaluating the success of mobile apps and its potential of gaining profits.

To fulfill these aims, the App recommends the users a series of exercise routines or gives the user a monthly plan based on the "fitness level" and goals. It specifies each exercise routine, length of time, purpose (e.g., which part of body one set of routine is supposed to work on), and the calories it will burn. Once the user finishes a day's exercise (or any other exercise), the achievement will show up in various visual forms - numbers, diagrams, circles, and accumulated levels/grades (See Fig. 1). This makes one of the central 


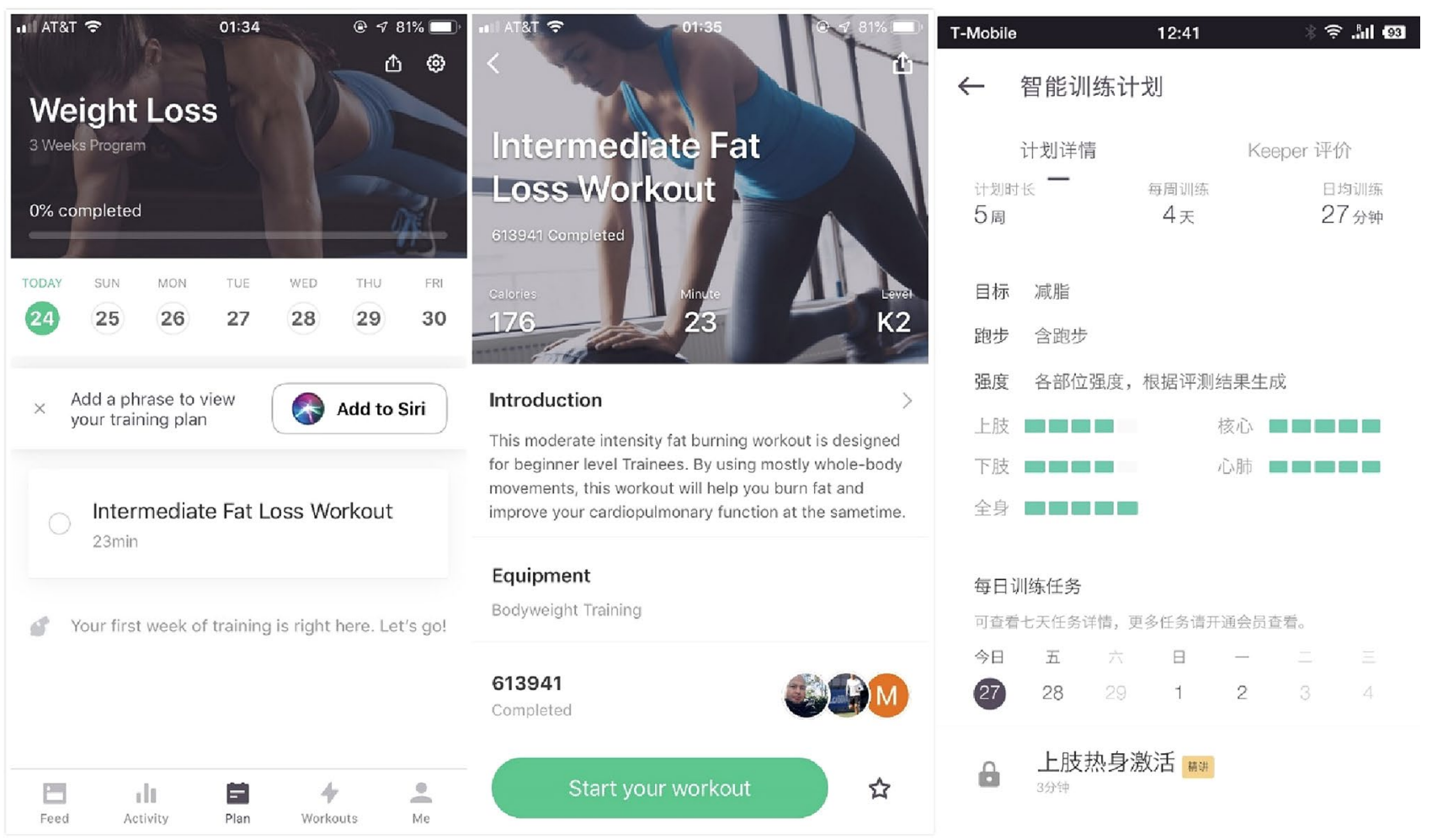

Fig. 2 Exercise plan. The left shows the "personalized plan" in a week's span and one routine for the day. For any routine, it shows an introduction, equipment, and how popular it is among users. The time and calories are the core elements, while there's a description of the "purpose" of this workout routine. However, terminologies are there; users do not have to understand them, and some are confusing

features of this app: a display of aggregated, quantified data that breaks down and visualizes the user's progress, thus help him/her "understand" what's going on with frequent self-reflection. For users, this form of presentation is clear, number-driven (by offering an "objective" knowledge of a subjective experience of fitness) and visually pleasing. Still, in this way, it leads to the scheme of self-surveillance in a nicely designed outfit.

"I pay a lot of attention to the numbers... I'll take my weight twice a day. I'll pay attention to the exercise time, 40 min per day... When I take a big meal, then I feel guilty about it, I'll take the most' intensive', calorie-burning exercise on the App...Yes, I feel better afterward." (\#11, female, in her $20 \mathrm{~s}$ ).

A personalized "plan" encourages daily and constant interactions from the users (See Fig. 2). The newest update in the fall of 2019 has set the "check-in" ${ }^{8}$ as one of the most

\footnotetext{
$\overline{8}$ In Chinese, the term “check-in” is fashioned as “打卡”, originating from the action that one goes to work and inserts the punchcard for recording the presence. It derives a broader meaning of doing something compulsory, or signifying "something has been done" without
}

due to bad translation. The right is how the training plan looks like in the Chinese version. The day-to-day routines are at the bottom, while the overall length (5 weeks, four days per week, 27 min per day) and goals, running and intensity are briefly displayed. The diagram shows how this plan enhances specific parts/capacity of the body (upper limb, lower limb, whole body, core, and cardiovascular)

visible features displayed on the user's home screen and during the interaction. It encourages users to participate in some daily activities for a certain goal while marking their participation with a "streak" on a calendar that shows continuous engagement.

The overall design is quantified and categorized based on one's performance and level of engagement. It translates into one's fitness status and encourages users to practice on a daily basis. The intentionality of this part of the design encourages constant "attention" and subsequent self-reflection in the form of "self-management" based on those evaluations and feedback. The intentionality channels the way the attention is given,

Through the intentionality of the design, the App creates and directs the user's experience of exercises ("feel better" when numbers are fulfilled), and even shapes one's perception of fitness (fitness or health is directly connected

\section{Footnote 8 (continued)}

describing further engagement, e.g. travelling to a tourist spot and taking a picture at the most famous place. 
with body metrics and fitness performances, presented in graphs). The design quantifies fitness levels and status, and at the same time, quantifies and interprets engagement as closely connected with fitness. Users interact with this part of the design with a sense of achievement by associating numbers to their life events. The majority of interviewees showed some form of these hermeneutic relations of meaning-making: fitness means to achieve and to excel to be the more motivated and active self, when this "self" could be monitored, quantified and displayed-a "transformation of perception." (Verbeek 2005: 130).

"It is a great incentive [to look at the numbers] ... I'll set my goal, and see how much I should do every day to achieve that. I can discipline and supervise myself." (\#9, male, in his $30 \mathrm{~s}$ ).

"I make summary every week and month...It shows me exercise time in each week and each month...last year it generated a picture of your annual achievement." (\#16, female, in her 30 s)

This hermeneutic relation mediates their experience and perception in a cultural context of "selfhood." As Lupton has argued, this presentation reflects the creation of a "data assemblage" (Lupton 2016). Designs and algorithms play a part in structuring the interaction and information on our devices, affecting our understandings of measurable health-encouraging the perfection of a specific body part by displaying numbers and achievements. ${ }^{9}$ Users interact with the visual and numerical display and presentation by a conscious meaning-making of self-tracking: recording, checking-back, and sharing on social media, expanding the realm of the so-called "dataveillance" of mundane life (van Dijck 2014). In this way, the App builds hermeneutic relation with the users, which co-shapes the perceptions of self-governed, self-surveilled health with extensive engagement. Also, The hermeneutic relations are gradually built-in and reinforced in some patterns of interactions. Users take a clear message from the App's contents and how the contents are displayed. For example, opening the app means checking with today's "duty" (plan) and seeing the current status of one's physical body - whether one has reached a goal, finished a commitment, or made progress. It will show visual progress as one starts and finishes each day's routine by filling out a circle. The hermeneutic relations between the users and the App automize the perceptual processes (as a "gestalt") and compose a version of reality for the users to take.

\footnotetext{
$\overline{9}$ For example, a set of exercise usually consists of a series of routines (such as push-ups, squats, or running distance), each specifically emphasizing the training of a body part or an aspect of fitness (e.g. endurance, strength, etc.).
}

"It automatically notifies you your physical activity. It will tell you which day you exercise or walk less in the past week which you could reflect on." (\#7, male, in his $40 \mathrm{~s}$ )

"I will 'check-in' after each exercise. It is a record of achievement; it makes me feel good, as I've accomplished something." (\#2, female, in her $20 \mathrm{~s}$ )

"(the automatically generated) running track is awesome, amazing. I'll save it and occasionally look back and reflect on it." (\#16, female, in her $30 \mathrm{~s}$ )

As I have written earlier, some users do "feel" better when they recognize what they have done, but this "feeling" comes more or less from the interpretation of achievement (hermeneutics). ${ }^{10}$ The seemingly mindless interactions with design features are built on these meanings. By designs such as giving user audio instruction during exercise (a female voice saying "come on!"), notifying daily plans with "push notifications," and pop-outs with sound and visual effects, the App provides a "nudge" (Lupton 2014) to user's self-surveillance behaviors as a "persuasive technology" (Fogg 2002). But only when the context of selfhood-a "better self" and "improvement" of self-are culturally and socially embedded in the user's perception are such persuasive designs persuasive rather than disturbing. As a form of personal "performing data" (Gill 2017), the numbers generated and logged in this technology reveals not only one's fitness status of a user but also a hermeneutically interpreted self in various material and embodied ways. It corresponds with the "biocultural discourse" (Rail 2012) in self-management and the making of selfhood that one relates to their own bodies in instrumentalist and economic vocabularies, in which better numbers are more desirable. Technology helps to record and regulate the user's practices in a quantified, user-friendly manner so that one could reach the ideal of being healthy and fit with the help of quantified, "objective" knowledge about bodily fitness. This knowledge, in turn, shapes user's subjective feelings and experiences.

Technologies and users help to shape each other in myriad ways, and such "intentionality" needs to be located in human-technology associations, as Verbeek (2011) argues as "hybrid character of humans and technologies." A broader concept of "hermeneutic strategy" (Rosenberger 2011) an interpretive framework in a certain social setting that certain meanings are more likely to be addressed and reinforced. It relates to the culturally historically sedimented procedures

\footnotetext{
${ }^{10}$ However, as the wearable technology moves forward, users are envisioning such as future when the inner, physical "self" would be bounded through more numbers and metrics on display; as seeing those numbers becomes a way of reading one's health and fitness without realizing the existence of technology. It is not yet mediating an embodiment relationship, but would march towards that.
} 
that bear upon the interpretation of a technological artifact. This framework can sometimes be co-constructed through both the design and use, a process of "intersubjectivity" (Secomandi 2018), which is culturally situated.

Therefore, the intentionality cannot always be reduced to what was explicitly delegated to the technology by its designers or users-a wider meaning-generating context should be taken into account as "multistable" relationships between technology and humans are simultaneously formed during interactions.

\subsection{Your health, my life: the multistability of technology}

You need to become self-disciplined and become part of the knowledge scheme in which fitness and health are measured by numbers on the mobile screen-however, not everyone buys into the scheme. There are constant negotiations between an ideal of "healthy-lifestyle" and real-life situations, sometimes with tension.

"I was looking for a way to get out of [a bad] situation while doing fitness was kind of easy. Just 10-15 min of exercise could boost myself up a bit." (\#11, regular tracker, female, in her $20 \mathrm{~s}$ ).

"Exercise for me is like tidying up the room...if I'm upset today, but I still have some energy left, then I'll spend 30 min to make me feel like achieving something." (\#20, casual user, female, in her $20 \mathrm{~s}$ ).

People feel like they were not achieving their potentialin their jobs, school, social life, or life situations that are not as quantifiable as doing a 30-min workout. The App offers immediate results and, over time, physical results as well. The mental aspect of exercise is not addressed in the App (at least in an apparent way). Nevertheless, users still make meaning from the quantifiable numbers for themselves.

Some users only view the App as a simple tool and reference point of fitness, but not as well-rounded lifestyle guidance (which the App pictures for them). Nevertheless, they could still interpret and associate the technology with their own context of life. As Verbeek (2005) addresses, the "multistability" of technology allows different interpretations to form during a user's interaction with the technology, and those interpretations and meanings should stand in the social context of technological experiences. ${ }^{11}$ The design

\footnotetext{
11 There are some nuances regarding to the term "multistability." Rosenberger and Verbeek (2015) pointed mainly to the various versions of use and interpretations from the user's side, and the divergence from the designing ends to the various perceptual ends, such as the case of fire hydrants (See Rosenberger 2017a) and "anti-homeless design." (See Rosenberger 2017b) However, when Ihde (1999) uses this for the analysis of imaging technology in the sciences, he argues that a "converge" of variations can also occur-different technologies
}

features of the technology and intentionality wrapped in the experiencing trajectories might indicate some form of stability here, but multiple "stabilities" should be seen with not only the design features of technology (or the designer's intention) but the cultural and social tendencies of interpretation - and this can occur to other fitness technologies with different visual designs.

The reasons for not closely managing fitness activities might vary. Still, nearly all of the users would agree that a better, healthier life should be well-managed and quantified, and the barrier to that is one's lack of determination, which the App could not offer. Again, this interpretation is out of the user's own context, not the App's agenda.

"Make apps are marketed as 'bite-size' exercise...but many people say that even you do not need a lot of time doing exercise, you still need motivation, or you should be very self-disciplined." (\#12, regular tracker, male, in his $30 \mathrm{~s}$ ).

A lot of variation comes from the "reading" of information and numbers. Even if the numbers are decorated with narratives of fitness, some other meanings can also be associated in another context. Postphenomenologists have analyzed scientific images (Ihde 1986 and 2009; Rosenberger 2011), medical data (Moerenhout et al. 2019) and medical images (Forss 2012), which can be read in different ways or even generating conflicts. It is part of instrumentally mediated perception regardless of the claim of "objective knowledge." It involves sensory and hermeneutic perception and interrelations between the two, and the process is "too tangible" for a fixed, abstract concept (Forss 2012).

Although nearly half of the interviewees see the App more or less "like a tool" for references when they need some exercise in the absence of gyms or outdoor activities (as is also the case during covid-19), it does not mean that the App is "just a tool." It still bears meanings and knowledge regimes by quantifying certain aspects of fitness and interpreting it into values such as self-discipline and selfsurveillance, but the meanings reach users via technological mediation. The multistability noted here allows users with their own social and cultural context to act differently, constituting their own subjectivity with material objectivity. As Lupton and Smith (2018) argue, users can express their "agential capacities," where self-improvement and control can be translated while they enact their own tracking practices.

Previous STS scholars use the term "interpretive flexibility" such as Bijker and Pinch (1984) to describe the stage

\section{Footnote 11 (continued)}

can hold on to certain imagery artefact from various channels. Whyte (2015) echoes with the idea of "pivot.". 
that different people interpret the attributes of the technological design before the final design is agreed upon and closed. Similarly, multistability indicates an extent of flexibility, but instead of a sociological process of conflicting, shaping and settling, there isn't a final closure of any kind. The design itself indicates a certain degree or form of flexibility and will be potentially exploited by different groups according to their social and cultural context.

In this study, the casual users (who exercise less often and take the App more as a reference tool than a self-management companion) showed different interpretation from the frequent users (who engage with their plans and goals daily) - the interplay between their own life course and the design elements went differently. For an app that covers a wide range of users, its interface design would certainly play out differently in different users. The interactions between design elements and various cultural assumptions call for further investigation.

In sum, the App sets the ideal of health and marches to it by self-surveillance, imposing the virtue of "self-discipline"- this aligns with most of the users' perceptions about keeping fit. The extent of achieving that ideal varies among users who combine their own source of motivation with the convenience and accessibility that the App offers. The users bring their "hermeneutic strategy" that involves approaching the app through their own value, not only through the engagement expected by the app designers. People would take things they deem useful from the App, and receive health-related information in "bite-sizes," but can still be skeptical about some aspects of it. They negotiate a "better version of me" that is shaped by the designs, creating their own narratives with the help of technology, compromising the ideal and the reality.

\section{Conclusion, discussion and future research}

The popularity of fitness apps has changed the way people exercise and understand exercise-into something manageable and trackable, as well as a healthy "lifestyle," This change happens during the contextualized interaction between people and technological designs. In this paper, I have examined the use of fitness apps and its connections with the understandings and practices of fitness/health during the engagement with technological designs. The intentionality of the "flow" of interface design creates an experience in which users are being guided through or subscribed to a normalized body scheme. Through the hermeneutic mediations of technological designs, the concepts of health and fitness are interpreted as "achievements" and associated with meanings that connect self-improvement and selfdiscipline with health and fitness. The findings correspond with the sociological analysis of the "quantified self" and the trends of self-surveillance, but are in finer details and connect with the cognitive and behavioral aspects of user experiences with the technology.

The quantificational management of self is implemented through the users' interaction with design elements and technological mediations of hermeneutics, or even further with aspirations towards embodiment (with extensive use of wearable gadgets in the future). However, the multistability of technological mediation creates opportunities for the users to re-interpret the context and meanings of fitness and exercises even within the design framework, showing that the designed path is far from determined. The understanding of fitness and health with personal technologies gadgets is a mutual (or even collective, if the users are to be connected into a social network) process.

The changing concepts of fitness and personal healthwith the unprecedented detailed personal informatics and numbers available through various gadgets and serviceshighlight the challenge of traditional methodologies and theories of sociological and behavioral studies in revealing the relationship between technological artifacts and healthrelated practices. The nuance of direct experiences-through which users recognize the significance and form understandings-is crucial in studying the impact of technology on personal matters such as health and fitness. The introduction of postphenomenology into the analysis of fitness apps and self-quantification behaviors provides a distinctive way of investigating the process of technological interactions, which gives weight to the design elements and interfaces on the shaping of our understandings, behaviors and practices. It casts light on both the materiality (technologies, designs, interfaces, or even systematic schemes) and perception (contents, messages, cultures) and mediating processes that connect both parts. As Latour (2005, see also Mol 2010) proposes, the phenomenon should be placed in a network of actors (actants), both human and nonhuman, to delineate a comprehensive picture of human-technology relations and to see the mutual impact of technologies and ideas. Postphenomenology offers a tool, a knife sharp enough to open the hard surface of a phenomenon and to make sound explanations.

This paper has limitations that most case studies share: a tension between internal and external validity. For future sociological examinations, an extensive analysis should be made on a variety of population groups, not limited to the typical app users (young, urban, highly educated). It will show a wide range of engagements and diverse perceptions, raising more questions about personalized health technologies and their social implications. The postphenomenology approach can also lead to investigations on other health-related technologies, including wearables, monitoring devices and services like "virtual helpers." It will bring 
helpful tools and useful perspectives to such interdisciplinary studies that further reveal the technologically mediated connections of human and health systems. Besides, there should also be conversations between the design and use, which is on-going through data-collecting, user observation, and engagement in the fitness market. The design process would reveal priorities and values from the App makers and how that will have an impact on the experiences and outcomes of fitness apps.

Finally, the approaches in this study could shed lights on the fluid context of health in times of turbulence and change (like in a global pandemic), and how the change in the political economy would trickle down to daily life-what does fitness and health means to a quarantined life? Would the urge of working out indoors converge with the technological intentionality that embraces and encourages the urge? The new questions call for innovative perspectives and new methodological tools to observe and dissect these unprecedented changes and further interrogate what kind of health we want and what technology could do for us.

Acknowledgements The author thanks Dr. Robert Rosenberger and Dr. Jennifer Singh from Georgia Tech for their advice and contributions towards this paper.

Funding The Author receives no funding for this study.

Data availability All data and materials support the published claims and comply with field standards and ethical requirements.

\section{Compliance with ethical standards}

Conflict of interest The Author declares no conflict of interest.

\section{References}

Ajana B (2013) Governing through biometrics: the biopolitics of identity. Bakingstoke: Palgrave Macmillan

Ajana B (2017). Digital health and the biopolitics of the Quantified Self. Digital Health. https://doi.org/10.1177/2055207616689509

Ajana B (Ed.) (2018). Metric culture: ontologies of self-tracking practices. Emerald group publishing, Bingley

Alam SYR (2016). Promissory failures: how consumer health technologies build value, infrastructures, and the future in the present. Doctoral dissertation, University of California, San Francisco

Charmaz K (2006). Constructing grounded theory: A practical guide through qualitative analysis. Thousand Oaks: Sage

Choe EK et al (2014) Understanding quantified-selfers' practices in collecting and exploring personal data. In: Proceedings of the 32nd annual ACM conference pp 1143-1152, New York: ACM

Clarke AE, Shim JK, Mamo L, Fosket JR, and Fishman JR (2003). Biomedicalization: Technoscientific transformations of health, illness, and US biomedicine. Ame Soc Rev 161-194

de Boer B (2020) Experiencing objectified health: turning the body into an object of attention. Med Health Care Philos. https://doi. org/10.1007/s11019-020-09949-0
Didžiokaitė G, Saukko P, and Greiffenhagen C (2018). Doing calories: the practices of dieting using calorie counting app myfitnesspal. In Ajana, B (Ed.) Metric Culture. Emerald Publishing Limited, Bingley pp 137-155

Dumit J (2003) Is it me or my brain? Depression and neuroscientific facts. J Med Human 24(1-2):35-47

Fogg BJ (2002) Persuasive technology: using computers to change what we think and do. Ubiquity 2002(December):5

Forss A (2012) Cells and the (imaginary) patient: the multistable practitioner-technology-cell interface in the cytology laboratory. Med Health Care Philos 15(3):295-308

Foucault M (1988). Technologies of the self. In Foucault, M. Technologies of the self: a seminar with Michel Foucault 16-49

Foucault M (1990) The history of sexuality, vol 1. Vintage Books, New York

Foucault M (2012) Discipline and punish: the birth of the prison. Vintage Books, New York

Gill KS (2017) Hermeneutic of performing data. AI Soc 32(3):309-320

Gui X, Chen Y, Caldeira C, Xiao D, and Chen Y (2017). When fitness meets social networks: Investigating fitness tracking and social practices on werun. In proceedings of the $2017 \mathrm{CHI}$ conference on human factors in computing systems. ACM $1647-1659$

Ihde D (1986). Experimental phenomenology: an introduction. SUNY Press, Albany

Ihde D (1990). Technology and the lifeworld: From garden to earth. Indiana University Press, Bloomington

Ihde D (1999). Expanding hermeneutics. In hermeneutics and science. Springer, Dordrecht 345-351

Ihde D (2002). Bodies in technology. University of Minnesota Press 5, Minneapolis

Ihde D (2009). Postphenomenology and technoscience: the peking university lectures. SUNY Press, Albany

Kristensen DB, Prigge C (2018) Human/technology associations in self-tracking practices. In: Ajana B (ed) Self-tracking. Palgrave Macmillan, Cambridge, pp 43-59

Kristensen DB, Ruckenstein M (2018) Co-evolving with self-tracking technologies. New Media Soc 20(10):3624-3640

Latour B (2005). Reassembling the social, Oxford University Press, Oxford

Lomborg S, Thylstrup NB, Schwartz J (2018) The temporal flows of self-tracking: Checking in, moving on, staying hooked. New Media Soc 20(12):4590-4607

Lupton D (2012) M-health and health promotion: the digital cyborg and surveillance society. Soc Theory Health 10(3):229-244

Lupton D (2014), Self-tracking modes: reflexive self-monitoring and data practices. Available at SSRN: https://ssrn.com/abstr act $=2483549$. (Accessed on August 19)

Lupton D (2016). The quantified self. John Wiley and Sons, New York

Lupton D (2019). Data selves: more-than-human perspectives. John Wiley and Sons, New York Neff and Nafus: Cambridge

Lupton D, and Smith GJ (2018) 'A much better person': the agential capacities of self-tracking practices. In: Metric Culture: ontologies of practices. Bingley: Emerald Publishing Limitedself-tracking

Maloney AE, Mellecker R, Buday R, Gao Z, Hinkley T, Esparza L, Alexander S (2015) Fun, flow, and fitness: opinions for making more effective active videogames. Games Health J 4(1):53-57

Martin AL, and Kluckner VJ (2014). Player-centered design model for psychophysiological adaptive exergame fitness training for children. In Schouten B, Fedtke S, Schijven M, et al. (Eds.) Games for Health 2014: proceedings of the 4th conference on gaming and playful interaction in healthcare. Springer Vieweg, Wiesbaden. 105-109.

Mayring P (2004) Qualitative content analysis. Comp Qual Res 1(2):159-176 
Millington B (2016) Fit for prosumption: interactivity and the second fitness boom. Media Cult Soc 38(8):1184-1200

Millington B, Millington R (2015) "The datafication of everything": toward a sociology of sport and big data. Sociol Sport J 32(2):140-160

Moerenhout T, Fischer GS, Devisch I (2019) The elephant in the room: a postphenomenological view on the electronic health record and its impact on the clinical encounter. Med Health Care Philos. https ://doi.org/10.1007/s11019-019-09923-5

Mol A (2010) Actor-network theory: sensitive terms and enduring tensions. Kölner Zeitschrift für Soziologie und Sozialpsychologie. Sonderheft 50:253-269

Neff G, and Nafus D (2016). The Self-Tracking. MIT Press

Pinch TJ, Bijker WE (1984) The social construction of facts and artefacts: or how the sociology of science and the sociology of technology might benefit each other. Soc Stud Sci 14(3):399-441

Rabinow P (2010) Artificiality and enlightenment: from sociobiology to biosociality. Politix 2:21-46

Rail G (2012) The birth of the obesity clinic: confessions of the flesh, biopedagogies and physical culture. Sociol Sport J 29(2):227-253

Rosenberger R (2011) A phenomenology of image use in science: multistability and the debate over Martian gully deposits. Techné Res Philos Technol 15(2):156-169

Rosenberger R (2017a) On the hermeneutics of everyday things: or, the philosophy of fire hydrants. AI Soc 32(2):233-241

Rosenberger R (2017b). Callous Objects: designs against the homeless. $\mathrm{U}$ of Minnesota Press, Minneapolis

Rosenberger R, Verbeek PP (2015) A field guide to postphenomenology. In: Verbeek PP (ed) Rosenberger R. Postphenomenological investigations, Essays on human-technology relations, pp 9-41

Rowse LM (2015). Statistics of the self: shaping the self through quantified self-tracking, scripps senior theses. Paper 695. http://schol arship.claremont.edu/scripps_theses/695

Ruckenstein M, Schüll ND (2017) The datafication of health. Ann Rev Anthropol 46:261-278

Ruffino P (2018) Engagement and the Quantified Self: Uneventful Relationships with Ghostly Companions. In: Ajana B (ed) SelfTracking. Palgrave Macmillan, Cambridge, pp 11-25
Secomandi F (2017) Digital Images and Multistability in Design Practice. In: Botin L (ed) Postphenomenology and Media: Essays on Human-Media-World Relations. Lexington Books, Lanham, pp $123-143$

Secomandi F (2018) Service Interfaces in Human-Technology Relations. New ways in mediating techno-human relationships, Postphenomenological Methodologies, p 83

Tripathi AK (2017) Hermeneutics of technological culture. AI Soc 32(2):137-148

Van Den Eede Y (2015) Tracing the tracker: a postphenomenological inquiry into self-tracking technologies. In: Rosenberger R, Verbeek PP (eds) Postphenomenological Investigations: Essays on Human-Technology Relations. Lexington Books, Lanham, pp $143-158$

van Dijck J (2014) Datafication, dataism and dataveillance: big data between scientific paradigm and ideology. Surveill Soc 12(2):197-208

Verbeek PP (2001) Don Ihde: the technological lifeworld. The empirical turn, American philosophy of technology, pp 119-146

Verbeek PP (2005). What things do: philosophical reflections on technology, agency, and design. Penn State Press, University Park

Verbeek PP (2008) Cyborg intentionality: rethinking the phenomenology of human-technology relations. Phenomenol Cognit Sci 7(3):387-395

Verbeek PP (2011). Moralizing technology: understanding and designing the morality of things. University of Chicago Press, Chicago

Whyte KP (2015) What is multistability? A theory of the keystone concept of postphenomenological research. The Manhattan papers, Technoscience and postphenomenology, pp 69-81

Publisher's Note Springer Nature remains neutral with regard to jurisdictional claims in published maps and institutional affiliations. 